\title{
Clarifying the role of salvage radiotherapy
}

\author{
Joseph Chin, MD, FRCSC
}

See related article on page 500

n this issue of CUAJ, the Genito-Urinary Radiation Oncologists of Canada (GUROC) group addresses several very important issues relating to salvage radiotherapy following biochemical relapse after radical prostatectomy. ${ }^{1}$ With the published results from the EORTC (European Organisation for Research and Treatment of Cancer) $22911^{2}$ and SWOG (Southwest Oncology Group) 8794 (NCIC PR-2) ${ }^{3}$ studies and the recent update of the latter trial showing metastasis-free and overall survival benefit for adjuvant radiotherapy for pT3 disease, ${ }^{4}$ the questions relating to salvage radiotherapy deserve more discussion.

A fundamental issue is the attitude of urologists toward the use of local salvage radiotherapy. There is a wide range of opinions among practitioners in various countries about whether radiotherapy should be recommended for prostate-specific antigen (PSA) relapse after radical prostatectomy, with those in favour ranging from $13 \%$ to $91 \%$. A safe assumption is that the Canadian position is more moderate and intermediate. The dichotomy in practice pattern stems largely from the lack of evidence from randomized controlled trials.

The GUROC consensus meeting included a discussion on 2 retrospective multi-institutional analyses by Stephenson and colleagues ${ }^{5,6}$ that revealed some surprising results. The 6-year progression-free probability was $32 \%$ for all participants, and even the most unfavourable group (those with a Gleason score of $8-10$, negative margins or a serum PSA level doubling time of $<10$ mo, i.e., those with a high probability of distant metastatic disease) had a 1 in 5 chance of achieving sustained progression-free response provided the local salvage radiotherapy was delivered when the serum PSA level was less than $2.0 \mu \mathrm{g} / \mathrm{L}$. Remarkably, when such patients were treated when the serum PSA level was less than $0.5 \mu \mathrm{g} / \mathrm{L}, 41 \%$ were disease-free at 6 years. These findings have led to the very reasonable recommendation by the GUROC for much earlier referral for salvage radiation consideration, even for patients with high-risk features. Another potential consequence of earlier radiotherapy may be obviation or deferral of androgen ablation (demonstrated in the SWOG 8794, NCIC PR-2 adjuvant radiotherapy trial $^{3,4}$ ), with the inherent benefits of avoiding hormonal therapy.

Although multivariable analysis from the retrospective multicentre study suggested a benefit for neoadjuvant androgen ablation ${ }^{6}$ the role of adjunctive hormonal therapy with salvage radiotherapy remains unsettled. The upcoming MRC (Medical Research Council) (NCIC PR-13) study hopes to answer some of the questions, including the timing and duration of hormonal therapy. The trial design appears somewhat complicated and imposing at first glance, but is in fact very logical and straightforward, and by providing an opportunity for most radical prostatectomy patients to participate at some point in the variable clinical course, the MRC will hopefully maximize accrual and eventually provide some valuable answers.

Sia and colleagues referred to the potential role of endorectal magnetic resonance imaging in guiding and planning salvage radiotherapy. Another imaging modality is the ProstaScint (Cytogen Corporation) capromab pendetide scan. ${ }^{7}$ More promising would be the fusion or coregistration of ProstaScint with another imaging modality such as CT. ${ }^{8,9}$ Ongoing studies with newer imaging techniques will assist radiation oncologist with making decisions about and fine-tuning salvage radiotherapy for the patient with biochemical relapse after radical prostatectomy.

The recommendations by GUROC, with acceptance and participation from urologists, should clarify the role of salvage radiotherapy after radical prostatectomy.

From the Division of Urology, University of Western Ontario, and the Division of Urology, London Health Sciences Centre, London, Ont.

Competing interests: None declared.

\section{References}

1. Sia M, Pickles T, Morton G, et al. Salvage radiotherapy following biochemical relapse after radical prostatectomy: proceedings of the Genito-Urinary Radiation Oncologists of Canada consensus meeting. CUAJ 2008;2:500-7.

2. Bolla $M$, van Poppel $H$, Collette $L$, et al. Postoperative radiotherapy after radical prostatectomy: a randomised controlled trial (EORTC trial 22911). Lancet 2005;366:572-4.

3. Thompson IM Jr., Tangen CM, Paradelo J, et al. Adjuvant radiotherapy for pathologically advanced prostate cancer: a randomized clinical trial. JAMA 2006;29:2329-35.

4. Swanson $G$, Thompson IM, Tangen $C M$, et al. Adjuvant radiotherapy for pT3 prostate cancer: results of a randomized prospective clinical trial, 2008 update [presentation]. American Urological Association Annual Meeting; 2008 May 17-22; Orlando (FL).

5. Stephenson AJ, Shariat SF, Zelefsky MJ, et al. Salvage radiotherapy for recurrent prostate cancer after radical prostatectomy. JAMA 2004;291:1325-32.

6. Stephenson AJ, Scardino PT, Kattan MW, et al. Predicting the outcome of salvage radiation therapy for recurrent prostate cancer after radical prostatectomy. J Clin Oncol 2007;25:2035-41.

7. Kahn D, Williams RD, Manyak MJ, et al. 111 Indium-capromab pendetide in 
the evaluation of patients with residual or recurrent prostate cancer after radical prostatectomy. The ProstaScint Study Group. J Urol 1998;159:2041-6.

8. Ellis RJ, Zhou EH, Fu P, et al. Single photon emission computerized tomography with capromab pendetide plus computerized tomography image set co-registration independently predicts biochemical failure. J Urol 2008;179:1768-73.

9. Chin IL, Belhocine T, Bauman G, et al. Added value of SPECT-TC to Prostascint imaging for biochemical failure following definitive local prostate cancer therapy [abstract]. CUAJ 2008;2:314.

Correspondence: Dr. Joseph Chin, Division of Urology, London Health Sciences

Centre, 800 Commissioners Rd. E., London ON N6A 4G5; jchin@lhsc.on.ca

\title{
We welcome your comments on the journal and on specific articles.
}

\author{
All letters will be considered
} for publication in the journal.

Send your letters to the Editor-in-Chief at journal@cua.org

\section{CUAJ JAUC}

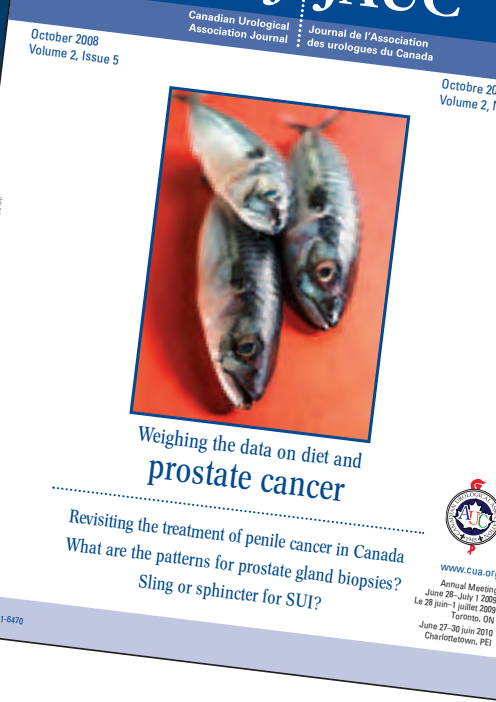

